\title{
HL-LHC physics with the ATLAS detector
}

\author{
M.P. Casado ${ }^{1}$ on behalf of the ATLAS collaboration. \\ ${ }^{1}$ Departament de Física, Universitat Autònoma de Barcelona, Institut de Física d'Altes Energies and Barcelona Institute of Science and \\ Technology, Spain.
}

Talk presented at 22nd High-Energy Physics International Conference in Quantum Chromodynamics (QCD 19), Montpellier, Languedoc, France, 02-05 July 2019

\begin{abstract}
Physics prospects for High-Luminosity LHC with the ATLAS detector are presented. The results presented here are based on updated and compiled projections published during 2019 in a Yellow Report. They include projections for Standard Model physics, Higgs physics, Beyond Standard Model physics, Flavor physics and QCD matter at high density. These proceedings give some highlights of the ATLAS physics potential in those areas ${ }^{2}$.
\end{abstract}

Keywords: HL-LHC physics, ATLAS, LHC

\section{Introduction}

The High-Luminosity LHC (HL-LHC) [1] is expected to start operation in 2026 (see Fig. 1). It will achieve an instantaneous luminosity of $7.5 \times 10^{34}$ $\mathrm{cm}^{-2} \mathrm{~s}^{-1}$ and will collect an integrated luminosity of $3000 \mathrm{fb}^{-1}$. With such beam conditions the average number of interactions per collision will be typically 200 , about one order of magnitude larger than during Run 2.

The ATLAS detector [2] will undergo a number of upgrades to cope with the HL-LHC running environment. The Data Acquisition (DAQ) and Trigger systems will improve their granularity and will have faster electronics achieving an output rate of $10 \mathrm{kHz}$. The Inner Tracker will be completely replaced using fully siliconbased technology, and its acceptance will be extended up to pseudorapidity equal to 4 . The calorimeters will also be upgraded with higher granularity and faster electronics. In the endcap region the electronics of the muon chambers will also be replaced to cope with the new

\footnotetext{
2 Copyright 2019 CERN for the benefit of the ATLAS Collaboration. Reproduction of this article or parts of it is allowed as specified in the CC-BY-4.0 license
}

conditions. Finally, High Granularity Timing Detectors are planned for the forward region of the ATLAS detector to perform timing measurements of charged tracks and help in the suppression of pile-up.

The performance of the ATLAS detector at HLLHC has been evaluated, either assuming similar detector performance to average Run 2 conditions or obtaining the estimates from pure MC analysis at particle level convoluted with expected object performance at HL-LHC. Theory uncertainties will dominate for many physics projections based on current extrapolations from existing Run 2 estimates. For example, the performance of the MV2 b-tagging algorithms in $t \bar{t}$ events is shown in Fig. 2 (extracted from [3]). It is expected to be improved by a factor of 2 within Run 2 detector acceptance, and the acceptance will be extended to $|\eta|<4$. The diphoton mass spectrum for $H \rightarrow \gamma \gamma$ has been estimated under HL-LHC conditions (see reference [4]) and it will not degrade with respect to Run 2 in an optimistic scenario even though the pile-up conditions increase considerably.

During 2019 ATLAS and CMS have compiled physics predictions for HL-LHC in 5 yellow reports: Working Group 1 (Standard Model) [6], Working Group 


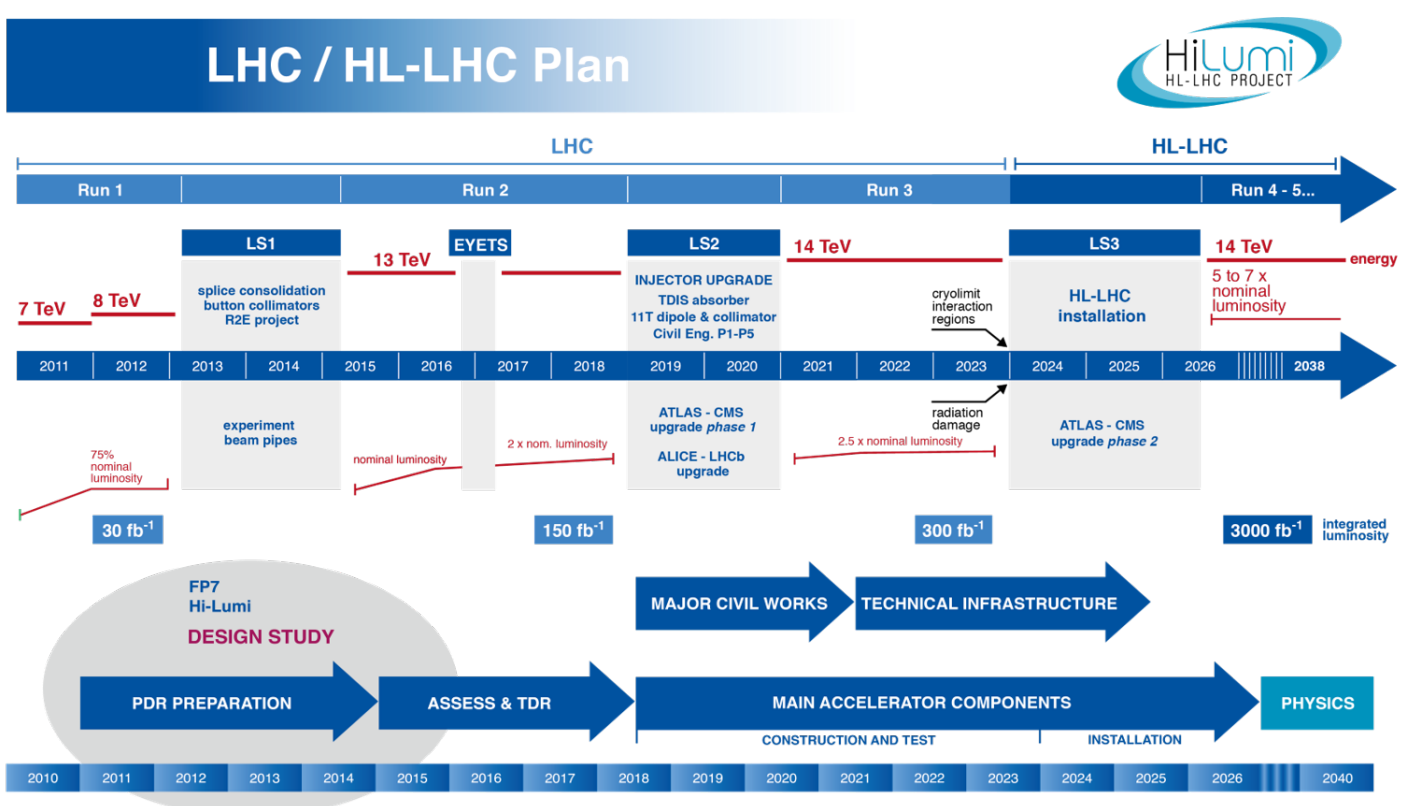

Figure 1: Timeline of the LHC baseline program and its upgrade phases, showing the energy of the collision (upper line -red) and instrumentation luminosity (lower lines -red) [1]. The second long shutdown (LS2) in 2019-20 will see the consolidation of the accelerator and the upgrade of the LHC Injectors. After the third long shutdown (LS3) in 2024-2026, the machine will be in the HL-LHC configuration.

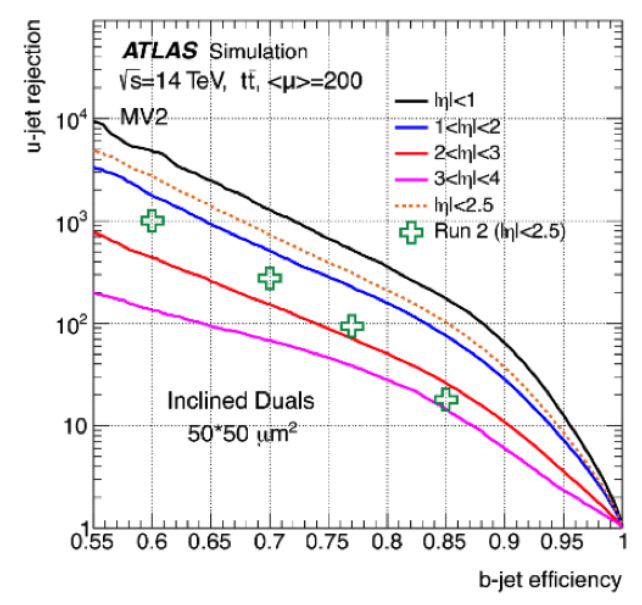

Figure 2: Performance of the MV2 b-tagging algorithm in $t \bar{t}$ events with 200 pile-up for the Inner Tracker layout [3]. The rejection of light jets for different $\eta$ regions is shown as a function of b-jet efficiency. Results are shown for $50 \times 50 \mu \mathrm{m}^{2}$ pixels, using digital clustering in the reconstruction. For comparison purposes, the performance for ATLAS during Run 2 with an average of 30 pile-up events is shown as crosses.

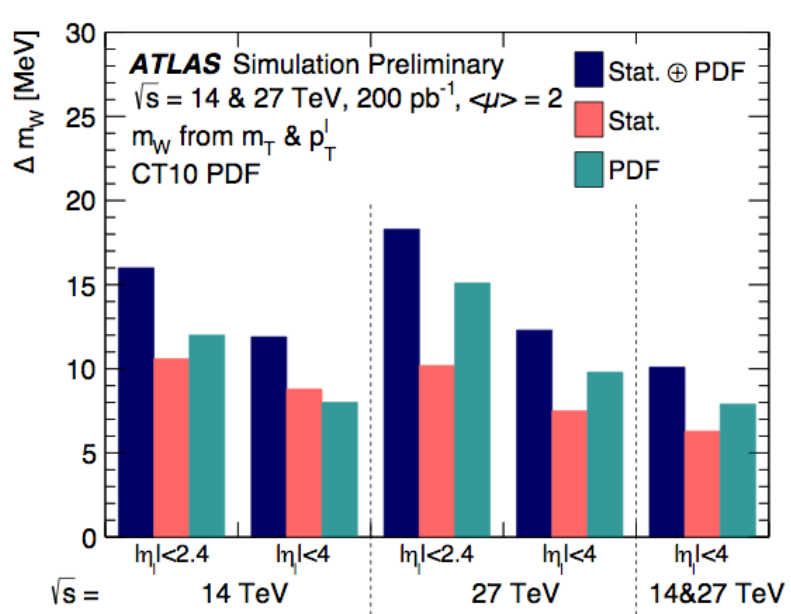

Figure 3: Measurement uncertainty for $m_{W}$ measurement at HL-LHC in different lepton acceptance regions and for different centre-of-mass energies, using CT10 PDF set and for $200 \mathrm{pb}^{-1}$ collected at each energy $[6,11]$. The numbers for $0<\left|\eta_{l}\right|<2.4$ correspond to the combination of the four pseudorapidity bins in this range. 


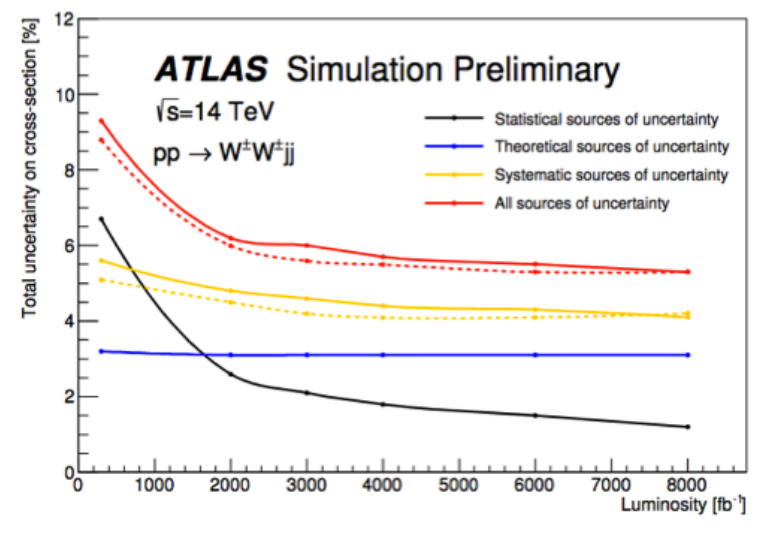

Figure 4: The estimated uncertainty of the EW $W^{ \pm} W^{ \pm}$cross section measurement as a function of the integrated luminosity [12]- [14].

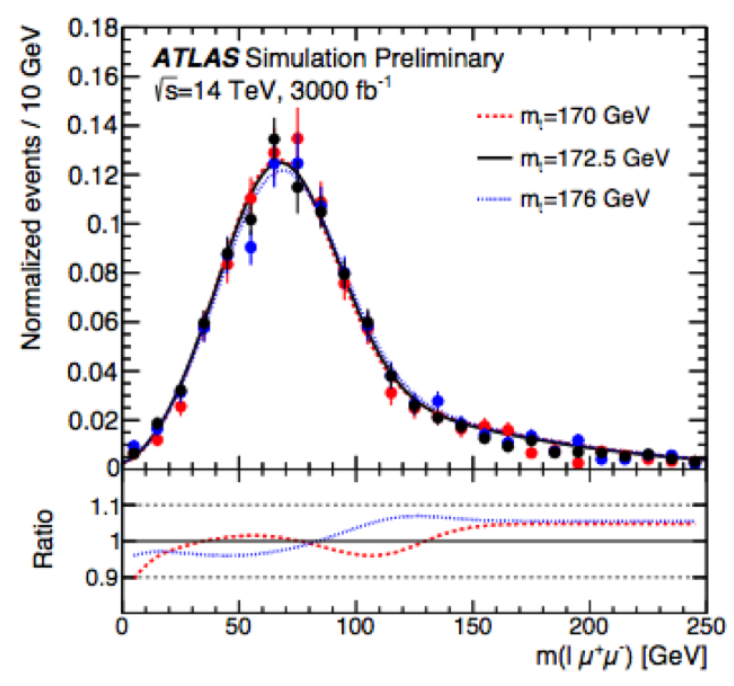

Figure 5: Template parametrization showing the sensitivity of $m\left(l \mu^{+} \mu^{-}\right)$to the input $m_{\text {top }}$ [15]. Each template (shown as points with uncertainties corresponding to statistical uncertainties) is overlaid with the corresponding probability density function (shown as lines) from the fit to templates. In addition, the lower panel shows ratios of the three fitted functions and the fitted function for $m_{\text {top }}=$ $172.5 \mathrm{GeV}$.
2 (Higgs) [7], Working Group 3 (Beyond the Standard Model) [8], Working Group 4 (Flavor) [9] and Working Group 5 (QCD matter at high density) [10]. These proceedings include some highlights from the 3 first reports.

\section{Standard Model Physics}

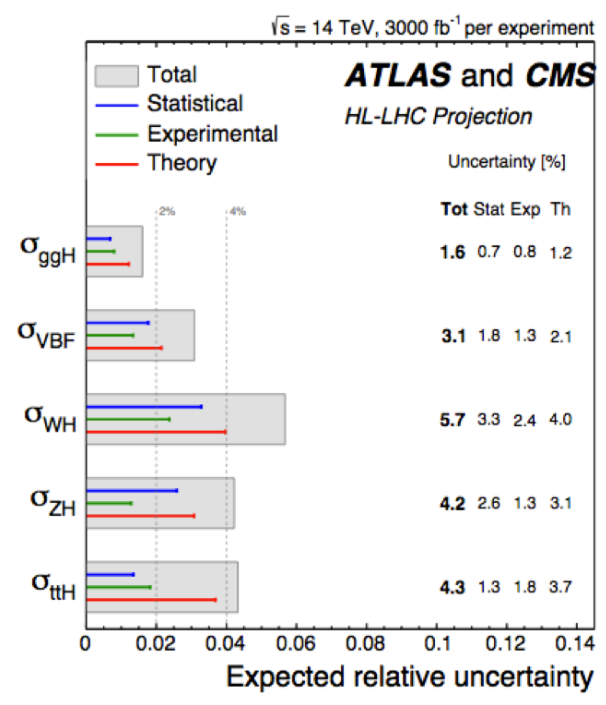

Figure 6: Summary plot showing the total expected $\pm 1 \sigma$ uncertainties for the HL-LHC projection on the per-production-mode cross sections normalised to the SM predictions for the combination of ATLAS and CMS extrapolations [7]. For each measurement, the total uncertainty is indicated by a grey box while the statistical, experimental and theory uncertainties are indicated by a blue, green and red line respectively. The numerical values are also reported.

The mass of the $\mathrm{W}$ boson will be measured with low pile-up collision data $[6,11]$. A total of $2 \times 10^{6} \mathrm{~W}$ boson events will be produced at $\sqrt{s}=14 \mathrm{TeV}$ with a luminosity of $5 \times 10^{32} \mathrm{~cm}^{-2} \mathrm{~s}^{-1}$ in one week. The understanding of PDF uncertainties will be critical and the extension of the inner detector to $|\eta|<4$ fundamental as can be seen in Fig. 3. The overall error can be decreased down to 10 $\mathrm{MeV}$.

Vector Boson Scattering processes, which are important tests of EW symmetry breaking mechanism, are expected to be observed at HL-LHC [12]- [14], with higher statistics and the instrumentation implemented in the forward region. Fig. 4 shows the estimated uncertainty on $W^{ \pm} W^{ \pm}$cross section measurement as a function of the integrated luminosity.

The mass of the top quark can be extracted from $t \rightarrow J / \psi\left(\mu^{+} \mu^{-}\right)$decays profiting from higher luminosity 
and larger acceptance of the inner detector (see Fig. 5 and reference [15]). The measurement will be based on the use of one lepton and $2 \mu$ 's in the final state and will exploit the sensitivity of the top quark mass to $m\left(l \mu^{+} \mu^{-}\right)$. The analysis will profit from high luminosity and larger acceptance of the inner detector. It will be limited by jet energy uncertainty scale on $m_{t}$ but $t \bar{t}$ modelling will also be relevant. The overall uncertainty is $0.50 \mathrm{GeV}$.

\section{Higgs Physics}

Figs. 6-9 show ATLAS and CMS combination plots for per-production-mode cross sections, branching ratios, coupling modifier parameters with respect to the SM $(\kappa)$ and ratio of coupling modifer parameters with respect to the SM $(\lambda)$. In most cases theory uncertainties are predominant. The theory correlations between experiments are neglected.

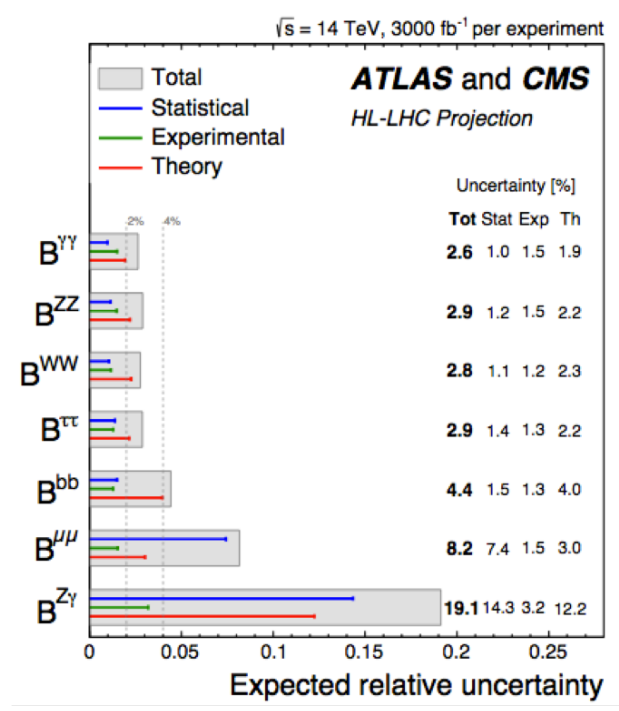

Figure 7: Summary plot showing the total expected $\pm 1 \sigma$ uncertainties for the HL-LHC projection on the per-production-mode branching ratios normalised to the SM predictions for the combination of ATLAS and CMS extrapolations [7]. For each measurement, the total uncertainty is indicated by a grey box while the statistical, experimental and theory uncertainties are indicated by a blue, green and red line respectively. The numerical values are also reported.

The self-coupling of the Higgs boson with respect to the SM $\left(\kappa_{\lambda}\right)$ will be extracted from HH mass spectrum and from the cross-section measurement. Figs.10 and 11 show the ATLAS and CMS combinations and the contribution from different analysis, respectively. An

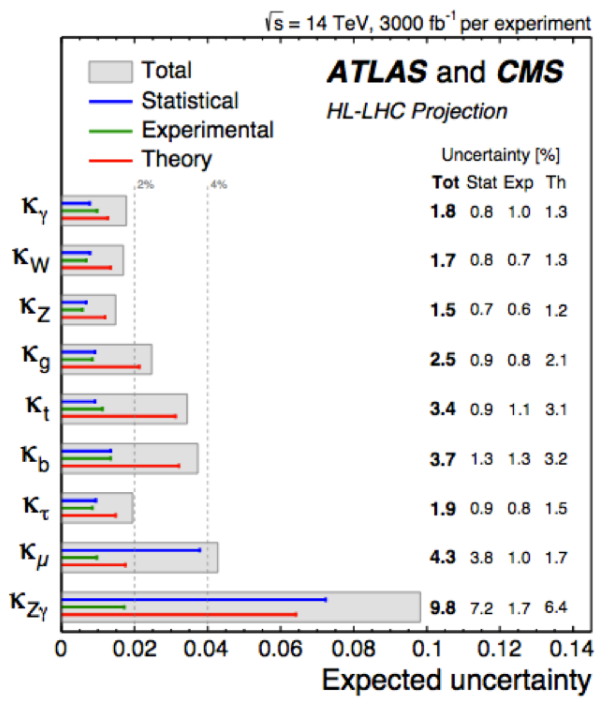

Figure 8: Summary plot showing the total expected $\pm 1 \sigma$ uncertainties for the HL-LHC projection on the coupling modifier parameters for the combination of ATLAS and CMS extrapolations [7]. For each measurement, the total uncertainty is indicated by a grey box while the statistical, experimental and theory uncertainties are indicated by a blue, green and red line respectively. The numerical values are also reported.

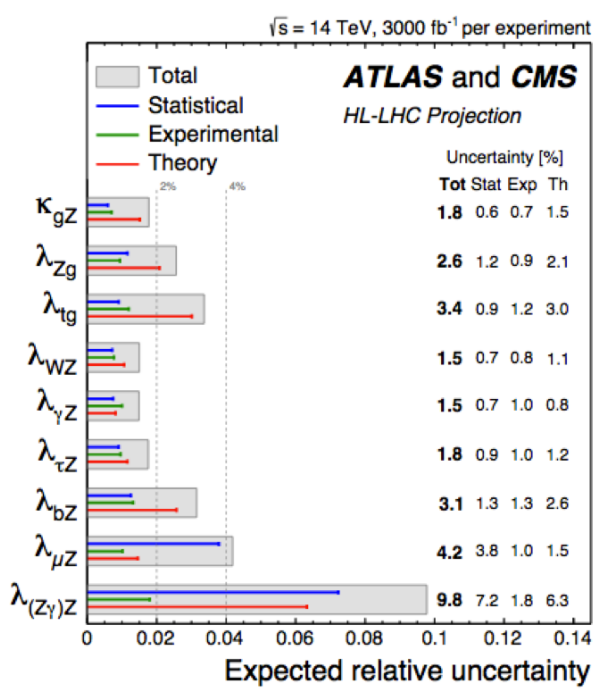

Figure 9: Summary plot showing the total expected $\pm 1 \sigma$ uncertainties for the HL-LHC projection on the ratios of coupling modifier parameters for the combination of ATLAS and CMS extrapolations [7]. For each measurement, the total uncertainty is indicated by a grey box while the statistical, experimental and theory uncertainties are indicated by a blue, green and red line respectively. The numerical values are also reported. 
overall significance of $4 \sigma$ can be achieved with the two experiments together.

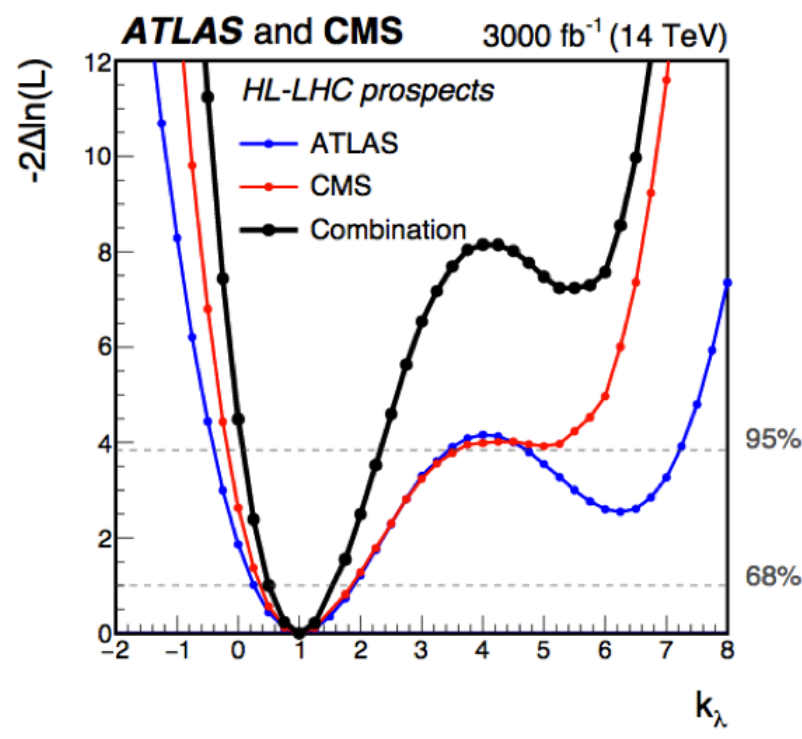

Figure 10: Minimum negative-log-likelihood as a function of $\kappa_{\lambda}$, calculated by performing a conditional signal+background fit to the background and SM signal [7]. The black line corresponds to the combined ATLAS and CMS results, while the blue and red lines correspond to the ATLAS and CMS standalone results respectively.

\section{Beyond the Standard Model Physics}

Dark Matter searches are becoming very relevant at LHC [17] in view of measurements obtained in other physics fields. Many physics interpretations are possible, namely scalar mediators, axial-vector mediators, SUSY, Axion Light Particles, Extra Dimensions, etc. In Fig. 13 the expected discovery contours for the axialvector simplified model are presented. The prediction is based on the analysis performed with $36 \mathrm{fb}^{-1}$ at $13 \mathrm{TeV}$ in which one energetic jet and $\mathrm{E}_{T}^{\mathrm{miss}}$ are required. The main systematic uncertainties are due to jet and $E_{T}^{\text {miss }}$ reconstruction.

Long lived particles will also be searched for at HLLHC [18]. Fig. 13 shows the projected sensitivity for the mass of a long lived gluino which hadronizes after production into an R-hadron, and then decays through a virtual squark into a pair of SM quarks and a neutralino. In the analysis high $\mathrm{E}_{T}^{\mathrm{miss}}$ and one displaced vertex are required. The main background is coming from hadronic interactions of SM particles with residual

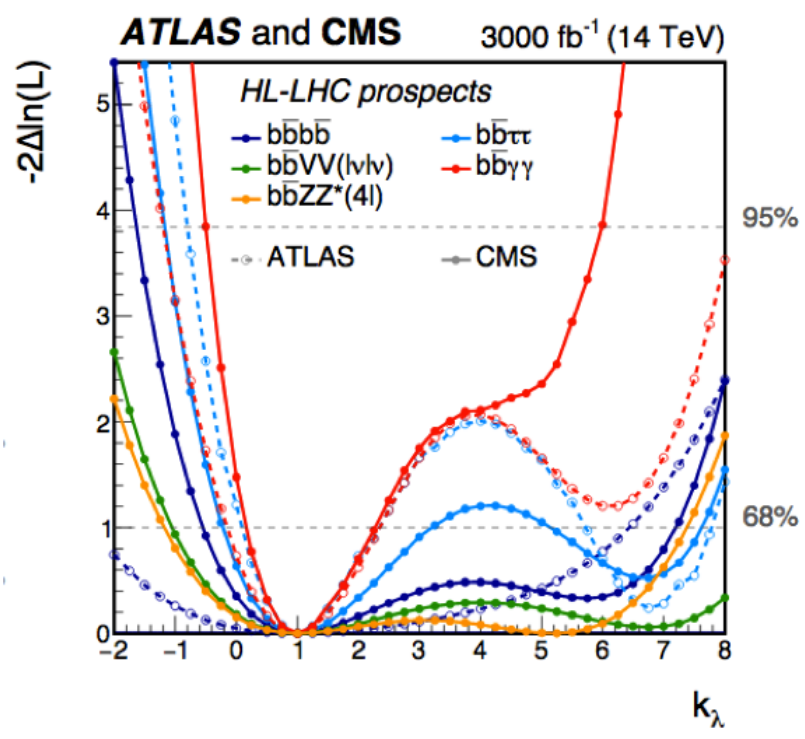

Figure 11: Minimum negative-log-likelihood as function of $\kappa_{\lambda}$, calculated by performing a conditional signal+background fit to the background and SM signal [7]. The different colours correspond to different channels, the plain lines correspond to the CMS results while the dashed lines correspond to the ATLAS results.

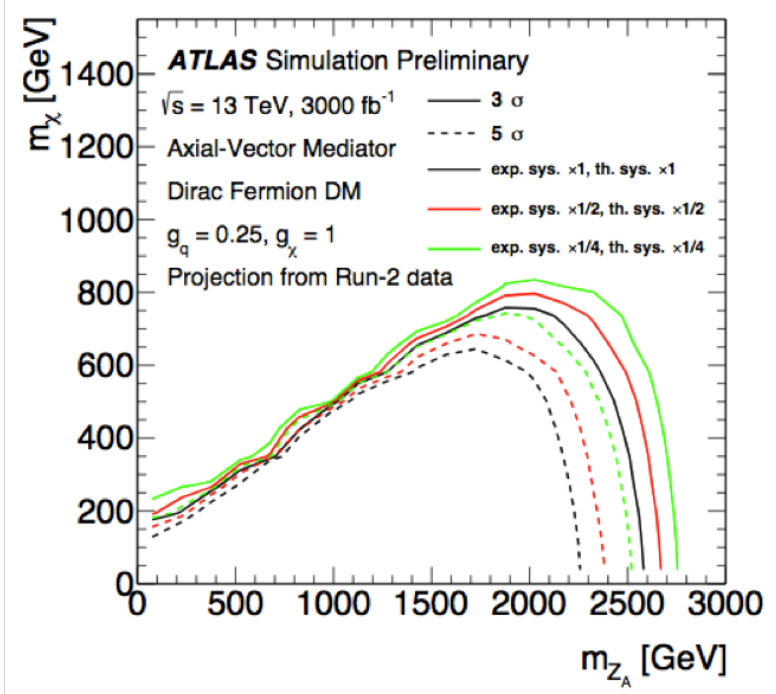

Figure 12: Expected $3 \sigma$ (solid) and $5 \sigma$ (dashed) discovery contours on the dark matter particle mass $\left(m_{\chi}\right)$ - axial vector boson mediator mass $\left(m_{Z A}\right)$ plane for the axial-vector simplified model with couplings $g_{\chi}=1$ and $g_{q}=0.25$, for a luminosity of $3000 \mathrm{fb}^{-1}$ [17]. Three countours are shown on the plot, corresponding to the three different systematic uncertainty scenarios: standard (black), reduced by a factor of 2 (red) and 4 (green). 
material. Gluino R-hadrons with masses up to $2.8 \mathrm{TeV}$ and lifetimes from 0.1 to $10 \mathrm{~ns}$ could be discovered.

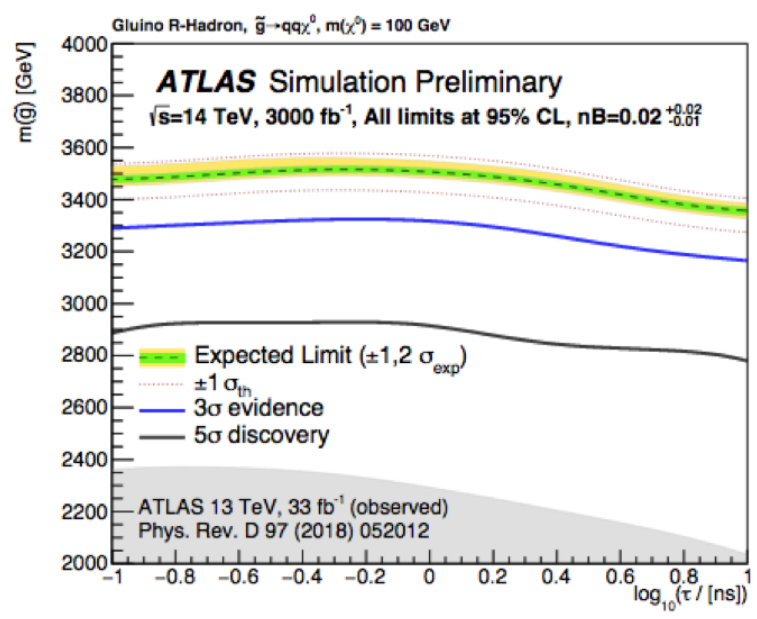

Figure 13: The projected sensitivity for the upper limit on the mass of a gluino $R$-hadron that can be observed with $3 \sigma$ and $5 \sigma$ confidence or excluded at $95 \% \mathrm{CL}$, as afunction of the gluino lifetime, for a background of $0.02_{-0.01}^{+0.02}$ events [18]. These results are valid for a gluino which decays to SM quarks and a stable neutralino with a mass of 100 $\mathrm{GeV}$. Results assume $3000 \mathrm{fb}^{-1}$ of collisions at $\sqrt{s}=14 \mathrm{TeV}$ collected with the upgraded ATLAS detector, and are compared to the observed ATLAS exclusion limits of $33 \mathrm{fb}^{-1}$ at $\sqrt{s}=13 \mathrm{TeV}$.

\section{Conclusions}

The High-Luminosity LHC constitutes a unique opportunity for many physics areas. Its potential has been updated and compiled recently in a set of publications [6]-[10] by the ATLAS and CMS collaborations. The estimates are either based on Run 2 extrapolations or based on pure MC analysis convoluted with expected object performances at HL-LHC. Theory uncertainties will be critical for many analyses and will need input from theory calculations and measurements from the experiments.

\section{References}

[1] G. Apollinari, I. Béjar Alonso, O. Brüning, M. Lamont, L. Rossi (Eds.), High-Luminosity Large Hadron Collider (HLLHC): Preliminary Design Report, CERN-2015-005.

[2] ATLAS Collaboration, The ATLAS Experiment at the CERN Large Hadron Collider, JINST 3 S08003 (2008).

[3] K. Einsweiler, L. Pontecorvo (Eds.), ATLAS Collaboration, Technical Design Report for the ATLAS Inner Tracker Pixel Detector, ATL-TDR-030, LHCC-2017-021.

[4] K. Einsweiler, L. Pontecorvo (Eds.), ATLAS Collaboration, Technical Design Report for the Phase-II Upgrade of the ATLAS LAr Calorimeter, ATL-TDR-027, LHCC-2017-018.
[5] ATLAS Collaboration, Expected performance of the ATLAS detector at the High-Luminosity LHC, ATL-PHYS-PUB-2019005 .

[6] P. Azzi, S. Farry, P. Nason, A. Tricoli, D. Zeppenfeld (Eds.), Standard Model Physics at the HL-LHC and HE-LHC, Report from Working Group 1 on the Physics of the HL-LHC, and Perspectives at the HE-LHC, arXiv:1902.04070.

[7] M. Cepeda, S. Gori, P. Ilten, M. Kado, F. Riva (Eds.), Higgs Physics at the HL-LHC and HE-LHC, Report from Working Group 2 on the Physics of the HL-LHC, and Perspectives at the HE-LHC, arXiv:1902.00134.

[8] X. Cid Vidal, M. D’Onofrio, P.J. Fox, R. Torre, K.A. Ulmer (Eds.), Beyond the Standard Model Physics at the HL-LHC and $H E-L H C$, Report from Working Group 3 on the Physics of the HL-LHC, and Perspectives at the HE-LHC, arXiv:1812.07831.

[9] A. Cerri, V.V. Gligorov, S. Malvezzi, J. Martín Camalich, J. Zupan (Eds.), Opportunities in Flavor Physics at the HL-LHC and $H E-L H C$, Report from Working Group 4 on the Physics of the HL-LHC, and Perspectives at the HE-LHC, arXiv:1812.07638.

[10] Z. Citron, A. Dainese, J.F. Grosse-Oetringhaus, J.M. Jowett, Y.J. Lee, U. Wiedemann, M. Winn (Eds.), Future physics opportunities for high-density $Q C D$ at the LHC with heavy-ion and proton beams, Report from Working Group 5 on the Physics of the HL-LHC, and Perspectives at the HE-LHC, arXiv:1812.06772.

[11] ATLAS Collaboration, Prospects for the measurement of the $W$ boson mass at the HL- and HE-LHC, ATL-PHYS-PUB-2018026.

[12] ATLAS Collaboration, Prospects for the measurement of the $W W$ scattering cross section and extraction of the longitudinal scattering component in pp collisions at the High-Luminosity LHC with the ATLAS experiment, ATL-PHYS-PUB-2018-052.

[13] ATLAS Collaboration, Prospect study of electroweak production of a $Z$ boson pair plus two jets at the HL-LHC, ATL-PHYSPUB-2018-029.

[14] ATLAS Collaboration, Prospective study of vector boson scattering in WZ fully leptonic final state at HL-LHC, ATL-PHYSPUB-2018-023.

[15] ATLAS Collaboration, Prospects for measurement of the top quark mass using $t \bar{t}$ events with $J / \psi \rightarrow \mu^{+} \mu^{-}$decays with the upgraded ATLAS detector at the High Luminosity LHC, ATLPHYS-PUB-2018-042.

[16] ATLAS Collaboration, Prospects for Dark Matter searches in mono-photon and $V B F+E_{T}^{\text {miss }}$ final states in ATLAS, ATLPHYS-PUB-2018-038.

[17] ATLAS Collaboration, Extrapolation of $E_{T}^{\text {miss }}+$ jet search results to an integrated luminosity of $300 \mathrm{fb}^{-1}$ and $3000 \mathrm{fb}^{-1}$, ATL-PHYS-PUB-2018-043.

[18] ATLAS Collaboration, Sensitivity of the ATLAS experiment to long-lived particles with a displaced vertex and $E_{T}^{\text {miss }}$ signature at the HL-LHC, ATL-PHYS-PUB-2018-033. 